\title{
Combined Perioperative Lapatinib and Trastuzumab in Early HER2-Positive Breast Cancer Identifies Early Responders: Randomized UK EPHOS-B Trial Long-Term Results
}

Nigel Bundred', Nuria Porta ${ }^{2}$, Adrian Murray Brunt ${ }^{3}$, Angela Cramer ${ }^{4}$, Andrew Hanby ${ }^{5}$, Abeer M. Shaaban ${ }^{6}$, Emad A. Rakha ${ }^{7}$, Anne Armstrong ${ }^{8}$, Ramsey I. Cutress ${ }^{9}$, David Dodwell ${ }^{10}$, Marie A. Emson ${ }^{2}$, Abigail Evans $^{11}$, Sue M. Hartup ${ }^{12}$, Kieran Horgan ${ }^{12}$, Sarah E. Miller ${ }^{2}$, Stuart A. McIntosh ${ }^{13}$, James P. Morden², Jay Naik $^{14}$, Sankaran Narayanan ${ }^{15}$, Jane Ooi ${ }^{16}$, Anthony I. Skene ${ }^{17}$, David A. Cameron ${ }^{18}$, and Judith M. Bliss ${ }^{2}$

\section{ABSTRACT}

Background: EPHOS-B aimed to determine whether perioperative anti-HER2 therapy inhibited proliferation and/or increased apoptosis in HER2-positive breast cancer.

Patients and Methods: This randomized phase II, two-part, multicenter trial included newly diagnosed women with HER2positive invasive breast cancer due to undergo surgery. Patients were randomized to: part 1 (1:2:2), no treatment (control), trastuzumab or lapatinib; part 2 (1:1:2) control, trastuzumab, or lapatinib and trastuzumab combination. Treatment was given for 11 days presurgery. Coprimary endpoints were change in Ki67 and apoptosis between baseline and surgery tumor samples (biologic response: $\geq 30 \%$ change). Central pathology review scored residual cancer burden (RCB). Relapse-free survival (RFS) explored long-term effects.

Results: Between November 2010 and September 2015, 257 patients were randomized (part 1: control 22, trastuzumab 57,

\section{Introduction}

The human epidermal growth factor receptor 2 (HER2) is a tyrosine kinase receptor amplified or overexpressed in $15 \%$ to $20 \%$ of breast cancers. HER2 lacks a specific ligand, and signaling occurs after the formation of heterodimers with HER1 and HER3 (1). Targeting this pathway improves outcomes for patients with HER2-positive breast cancer. Trastuzumab interacts with the extracellular domain of the HER2 protein to inhibit its function $(1,2)$, but the mechanism of action

'Manchester University NHS Foundation Trust and University of Manchester, Manchester, United Kingdom. ${ }^{2}$ The Institute of Cancer Research, Clinical Trials and Statistics Unit, London, United Kingdom. ${ }^{3}$ University Hospitals of North Midlands and Keele University, United Kingdom. ${ }^{4}$ The Christie Pathology Partnership, Manchester, United Kingdom. ${ }^{5}$ Leeds Institute of Medical Research at St. James's, Leeds, United Kingdom. ${ }^{6}$ Queen Elizabeth Hospital Birmingham and University of Birmingham, Birmingham, United Kingdom. ${ }^{7}$ University of Nottingham, Nottingham, United Kingdom. ${ }^{8}$ The Christie NHS Foundation Trust Manchester, United Kingdom. ' ${ }^{9}$ University of Southampton and University Hospital Southampton, Southampton, United Kingdom. ${ }^{10}$ Nuffield Department of Population Health, University of Oxford, Oxford, United Kingdom. ${ }^{11}$ Poole Hospital NHS Foundation Trust, United Kingdom. ${ }^{12}$ St James's University Hospital, Leeds, United Kingdom. ${ }^{13}$ Queen's University Belfast, Belfast, United Kingdom. ${ }^{14}$ Mid Yorkshire NHS Hospitals Trust, United Kingdom. ${ }^{15}$ University Hospitals of North Midlands, Stoke-on-Trent, United Kingdom. ${ }^{16}$ Royal Bolton Hospital, Manchester, United Kingdom. ${ }^{17}$ University of Southampton, Southampton, United Kingdom. ${ }^{18}$ University of Edinburgh Cancer Research Centre, Institute of Genetics and Cancer, Western General Hospital, Edinburgh, United Kingdom. lapatinib 51; part 2: control 29, trastuzumab 32, combination 66). Ki67 response was evaluable for 223 patients: in part 1 Ki67 response occurred in 29/44 (66\%) lapatinib versus $18 / 49$ (37\%) trastuzumab $(P=0.007)$ and $1 / 22(5 \%)$ control $(P<0.0001)$; in part 2 in $36 / 49$ (74\%) combination versus $14 / 31(45 \%)$ trastuzumab $(P=0.02)$ and $2 / 28(7 \%)$ control $(P<0.0001)$. No significant increase in apoptosis after 11 days was seen in treatment groups. Six patients achieved complete pathologic response (pCR, RCB0) and 13 RCB1, all but two in the combination group. After 6 years median follow-up, 28 (11\%) had recurrence and 19 (7\%) died. No recurrences or deaths were observed among patients who achieved a pCR. Ki67\% falls $\geq 50 \%$ associated with fewer recurrences $(P=0.002)$.

Conclusions: Early response after short duration anti-HER2 dual therapy identifies cancers dependent on the HER2 pathway providing a strategy for exploring risk-adapted individualized treatment de-escalation. is incompletely understood. Lapatinib blocks the HER1/2 internal tyrosine kinase domain and inhibits proliferation of HER2-positive cancers (3) as shown in a small preoperative trial (4).

Changes in proliferation biomarkers, including Ki67, predict clinical response and long-term outcome after 2 weeks of endocrine therapy in estrogen receptor (ER)-positive breast cancer (5-7). Incompletely excised breast cancers requiring re-excision within 48 days of surgery showed a significant increase in proliferation if they were HER2-positive, but not if they were HER2-negative (8). Preventing

Note: Supplementary data for this article are available at Clinical Cancer Research Online (http://clincancerres.aacrjournals.org/).

D.A. Cameron and J.M. Bliss share senior authorship.

${ }^{\dagger}$ Deceased.

Corresponding Author: Nigel Bundred, Department of Academic Surgery, Manchester University Foundation Trust, 2nd Floor Education and Research Centre, Manchester, M23 9LT, United Kingdom. E-mail: nigel.bundred@manchester.ac.uk

Clin Cancer Res 2022;XX:XX-XX

doi: 10.1158/1078-0432.CCR-21-3177

This open access article is distributed under the Creative Commons Attribution License 4.0 International (CC BY).

C2022 The Authors; Published by the American Association for Cancer Research 


\section{Translational Relevance}

- In a randomized trial of 257 patients with HER2-positive breast cancer, lapatinib (alone 66\% or in combination 74\%) for 11 days produced higher Ki67 response rates than trastuzumab alone $(37 \%-45 \%)$ or control $(5 \%-7 \%)$.

- Combination treatment achieved a pCR or RCB1 in $26 \%$ cancers.

- After median follow-up of 6 years, perioperative falls in Ki67\% of $50 \%$ or more were associated with a lower relapse rate than smaller or no decrease in Ki67.

- Early response to therapy identifies cancers dependent on the HER2 pathway, allowing individualization of treatment.

these early changes provides a rationale for window-of-opportunity studies investigating response to short-term treatment, enhancing prospects for personalizing medicine by identifying tumors sensitive to anti-HER2 therapy (without added chemotherapy).

The Effect of Perioperative Anti-HER2 therapy on Early Breast Cancer Study - Biological phase (EPHOS-B) was designed to assess whether either single-agent lapatinib or trastuzumab given as perioperative treatment had effects on Ki67 and/or apoptosis compared with no anti-HER2 therapy prior to surgery (part 1). Emerging evidence from the NeoSphere trial (9) on the safety and efficacy of combination anti-HER2 therapy led to a protocol amendment, enabling patient allocation between control, trastuzumab alone, or the combination of lapatinib and trastuzumab (part 2). Although the primary biological endpoint reported here is a short-term biomarker, its presentation is accompanied by analyses illustrating impact on 5-year disease outcomes and exploratory analysis associating response with stromal tumor-infiltrating lymphocytes (TILs).

\section{Materials and Methods Design and patients}

EPHOS-B (NCT01104571) was a phase II, open-label, randomized, UK multicenter trial conducted in two parts, in newly diagnosed women with HER2-positive invasive breast cancer due to undergo surgery within 28 days. Patients had to be willing to undergo adjuvant chemotherapy and trastuzumab postsurgery as per standard of care and provide written informed consent for participation and donation of tissue and blood samples. Patients with significant cardiac abnormalities were ineligible. Baseline left ventricular ejection fraction (LVEF) $\geq 55 \%$ was required for trial entry. Full selection criteria are to be found in Appendix 1. The trial was conducted in accordance with Good Clinical Practice Guidelines and the Declaration of Helsinki.

\section{Procedures}

In part 1 , patients were randomized (1:2:2) to receive no perioperative treatment (control), trastuzumab alone, or lapatinib alone. In part 2, patients were allocated (1:1:2) to control, trastuzumab, or lapatinib and trastuzumab combined. Treatment commencement date was agreed on prior to randomization as 11 days $(+2 /-1)$ before the scheduled surgery. Treatment allocation was open-label, computergenerated, and centrally performed via telephone to the trials unit. In part 1, permuted blocks (up to size 12) stratified by center were used; in part 2, minimization with a random element and center as balancing factor was adopted to avoid imbalance given the smaller than expected number of patients randomized per center.

Trastuzumab (alone or in combination) was given intravenously before surgery on days 1 and 8 at an accelerated loading schedule dose of $6 \mathrm{mg} / \mathrm{kg}$ (to achieve faster steady-state levels of therapeutic efficacy; ref. 10) and one dose of $2 \mathrm{mg} / \mathrm{kg}$ was given after surgery between days 15 and 19. In part 1 , lapatinib was given at a dose of $1,500 \mathrm{mg}$ /day orally continuously for 28 days including the day of surgery. In part 2, when combined with trastuzumab, the lapatinib dose was $1,000 \mathrm{mg} / \mathrm{day}$ orally for 28 days.

Definitive surgery was according to local practice and patient choice. If nodal involvement was identified preoperatively, axillary clearance was the standard treatment. Adjuvant treatment was as per local policy and not influenced by EPHOS-B allocated treatment (see Appendix 1). Patients were followed up for cardiac toxicities and disease outcome every 6 months for 2 years after randomization, then annually.

\section{Assessment of biomarkers}

Formalin-fixed, paraffin-embedded (FFPE) tumor blocks from diagnostic core biopsy and surgical specimens were centrally assessed for quality and tumor content, and analyzed for Ki67 and activated caspase 3 (apoptosis) by IHC using methods described previously $(4,5,11)$. Hormone receptor status (ER and, when available, progesterone receptor $[\mathrm{PgR}]$ ) was locally evaluated by IHC: Allred (or Quickscore), percentage tumor cells, or $H$ score were recorded if available. The cut-off for positivity was $\geq 1 \%$ tumor cells, or Allred/Quickscore $\geq 3$. HER2 was evaluated locally and judged positive by IHC $3+$ score or FISH amplification $(12,13)$. FISH assessment was retrospectively repeated centrally. Central scoring of stromal TILs was conducted by specialist breast pathologists following international recommendations (14) on scanned $\mathrm{H} \& \mathrm{E}$ baseline and surgery slides.

Unexpectedly, a proportion of patients had insufficient tumor tissue in the surgical specimen for biomarker analysis. A review of pathology reports blinded to allocated treatment was undertaken to identify cases with evidence of potential tumor regression, and their pathology centrally assessed. Tumor bed sections at surgery were reviewed to confirm pathologic complete response (pCR) or, if the tumor were still present, to assess the two largest measurable spans of tumor, cellularity of the tumor within the tumor bed, and ductal carcinoma in situ (DCIS) within the tumor. Lymph node stage was recorded from pathology reports, and the size of the largest metastatic deposit measured. If there was detectable evidence of tumor response and nodal status was known, residual cancer burden (RCB) score and class [RCB0 (pCR); RCB1 (minimal residual disease); RCB2/3 (moderate or extensive residual disease)] were calculated (15). The remaining cases not selected for central pathology review were considered RCB2/3.

\section{Outcomes}

The coprimary endpoints were changes in Ki67 and in apoptosis, with biological response defined as a relative decrease in Ki67 of $>30 \%$, or an increase in apoptosis of $>30 \%$ between baseline and surgery (16). Secondary endpoints included relapse-free survival (RFS, time from randomization to local, regional, distant tumor recurrence, or death from any cause, with second primary cancers censored), overall survival, and safety. Exploratory endpoints included disease response at surgery, HER2 amplification by FISH, and changes in TILs during the perioperative period. 


\section{Statistical analysis}

The planned sample size $(N=250)$ assumed that biological response in Ki67 or apoptosis would be $\leq 5 \%$ in the control group compared with $>30 \%$ in the treatment groups and powered to detect $30 \%$ differences in response rate between treatment groups. With $2.5 \%$ one-sided type I error, $85 \%$ power for the treatment-control comparisons, and $>80 \%$ for between-treatment comparisons, 40 lapatinib (L, part 1), 55 control (C, part 1:20, part 2:35), 75 trastuzumab (T, part 1: 40, part 2: 35$)$, and 80 combination ( $\mathrm{T}+\mathrm{L}$, part 2) patients were required. Between-group comparisons were restricted to concurrently randomized patients: $L$ versus $C$ (part 1$), L$ versus $T$ (part 1$), T+L$ versus $\mathrm{T}$ (part 2), $\mathrm{T}+\mathrm{L}$ versus $\mathrm{T}$ (part 2 ), and $\mathrm{T}$ versus $\mathrm{C}$ (part 1 and part 2).

Perioperative change endpoints were analyzed on all patients who had paired biological data; patients who were found ineligible before starting any treatment were excluded. Surgical Ki67 and apoptosis scores in patients with breast pCR (regardless of nodal status) were excluded from the analysis. We conducted a sensitivity analysis on Ki67\% assuming such patients had a $0 \%$ score at surgery. Percentage changes by randomized treatment group were compared by MannWhitney tests. The proportion of patients responding in Ki67 and/or apoptosis analyses were compared using Fisher exact test. An alternative threshold of $>50 \%$ reduction (as used in the MAPLE trial; ref. 4 ) was also explored. The proportion of patients with pCR or RCB1 was described for each treatment group.

All randomized patients were included in the analysis of time-toevent endpoints, summarized by Kaplan-Meier estimates, and groups compared with log-rank tests. Association of perioperative biological changes with RFS was considered exploratory in nature; part 1 and part 2 were combined for this, and log-rank tests stratified by treatment group. Perioperative \%Ki67 decrease was categorized into decrease of $50 \%$ or more, $10 \%$ to $50 \%$ decrease; or no relevant decrease ( $<10 \%$ decrease or no decrease). Absolute Ki67 values were categorized following on from work in endocrine sensitive breast cancer (7): baseline and surgery Ki67 were high if $10 \% \leq$ or low if $<10 \%$, and combined into "high-high," "high-low," "low-high," or "low-low" categories. Patients with pCR or $0 \%$ breast cellularity were imputed a value of $0 \%$ at surgery and included in these analyses.

TILs were measured as a percentage (occupation of TILs in the tumor stromal surface area) and categorized into low $(\leq 20 \%)$ or high TILs (>20\%; ref. 17). Analysis of changes in TILs was restricted to patients with paired baseline and surgery data and no evidence of tumor regression at surgery (i.e., RCB2/3) to account for the lack of samples to perform analyses in pCR and patients in the RCB1 group. TILs were associated with trial outcomes, for which part 1 and part 2 data were combined.

A 5\% significance level was considered for treatment comparisons of primary and secondary endpoints and $1 \%$ for all other exploratory analyses. Stata (v13.0 or later) statistical software was used. Data cutoff for biomarker analyses was July 14, 2017; updated for 5-year outcomes on December 20, 2020. Further details of the methodology are available in the Appendix 1.

\section{Data availability}

Formal requests for sharing the data generated in this study will be considered with due regard given to funder and sponsor guidelines. Requests involving collaboration with the EPHOS-B Trial Management Group (TMG) are strongly encouraged. Requests are reviewed by the TMG and will be considered dependent on scientific merit, ethical considerations including patient consent, funding, resources, and alignment with the trial objectives. Data sharing are further approved by the Trial Steering Committee.

\section{Results}

\section{Patient disposition and baseline characteristics}

Two hundred fifty-seven patients were recruited from 21 UK centers; 130 entered part 1 between November 15, 2010, and July 29, 2013, and 127 entered part 2 between August 6, 2013 and September 10,2015 . Two patients (1\%) were found ineligible before starting treatment and excluded from the analysis of perioperative endpoints (Fig. 1). Overall, 172 patients (67\%) had ER-positive tumors, with a median tumor size of $2.2 \mathrm{~cm}$ (Table 1). Details of adjuvant treatment following surgery are provided in Appendix 2; with no differences between randomized groups in adjuvant treatment received.

\section{Disease response}

Forty of 255 patients showed evidence of potential tumor regression in the central review of pathology reports and underwent central RCB scoring; the remainder were considered RCB2/3. Although this analysis was originally unplanned, it became an essential component of the main trial analysis to inform impact of disease regression on the primary biomarker endpoints (informative censoring).

In part 1, 1/56 (2\%) pCR was observed in the trastuzumab group. In part 2, 1/32 (3\%) pCR occurred in the trastuzumab group, whereas in the combination group 4/65 (6\%) achieved pCR, and 13/65 (20\%) RCB1 were identified, including two-node positive patients who were node negative at surgery (Appendix 3). Two further combination treated patients who had scored RCB1 and RCB2/3 (due to nodal involvement) showed no residual disease in the breast $(0 \%$ breast cellularity). Among the 19 patients in the pCR or RCB1 group, 14 (74\%) had ER-positive tumors (Appendix 3). All but one (patient choice) received adjuvant chemotherapy as per local practice.

Before therapy, median (min-max) radiologic tumor size was $2 \mathrm{~cm}$ $(0.9-2.8)$ for patients who achieved pCR, $1.4 \mathrm{~cm}(0.5-4.5)$ for RCB1 patients, and $1.9 \mathrm{~cm}(0.1-10)$ for RCB2/3 patients. In a multivariable analysis in the combination group, only size of tumor was associated with observing pCR/RCB1 response (Appendix 3).

\section{Ki67}

Waterfall plots illustrating the range of percentage change in Ki67 observed in individual patients are presented in Fig. 2A and $\mathbf{B}$.

In part 1, 29/44 (66\%) patients taking lapatinib had a Ki67 response ( $\geq 30 \%$ reduction) compared with $18 / 49$ (37\%) patients taking trastuzumab $\left(P_{\mathrm{LvT}}=0.007\right)$ and $1 / 22(5 \%)$ patients in the control group. $\left(P_{\mathrm{LvC}}<0.0001\right)$. Median percentage change in Ki67 was $-43 \%$ (IQR $-68 \%$ to $-21 \%$ ) with lapatinib, $-14 \%$ (IQR, $-51 \%$ to $+6 \%$ ) with trastuzumab, and $+2 \%$ (IQR, $-9 \%$ to $+15 \%$ ) with control (Table 2 Appendix 4.1).

In part 2, 36/49 (74\%) patients in the combination group had a Ki67 response compared with $14 / 31(45 \%)$ patients in the trastuzumab group $\left(P_{\mathrm{T}+\mathrm{LvT}}=0.02\right)$ and $2 / 28(7 \%)$ patients in the control group $\left(P_{\mathrm{T}+\mathrm{LvC}}<0.0001\right)$. Median percentage change in Ki67 was $-49 \%$ (IQR $-78 \%$ to $-25 \%$ ) with combination, $-26 \%$ (IQR, $-46 \%$ to $-6 \%$ ) with trastuzumab and $-2 \%$ (IQR, $-20 \%$ to $+7 \%$ ) with control (Table 2 Appendix 4.1).

When combining part 1 and part 2, 32/80 (40\%) patients in the trastuzumab group had a Ki67 response compared with 3/50 (6\%) 


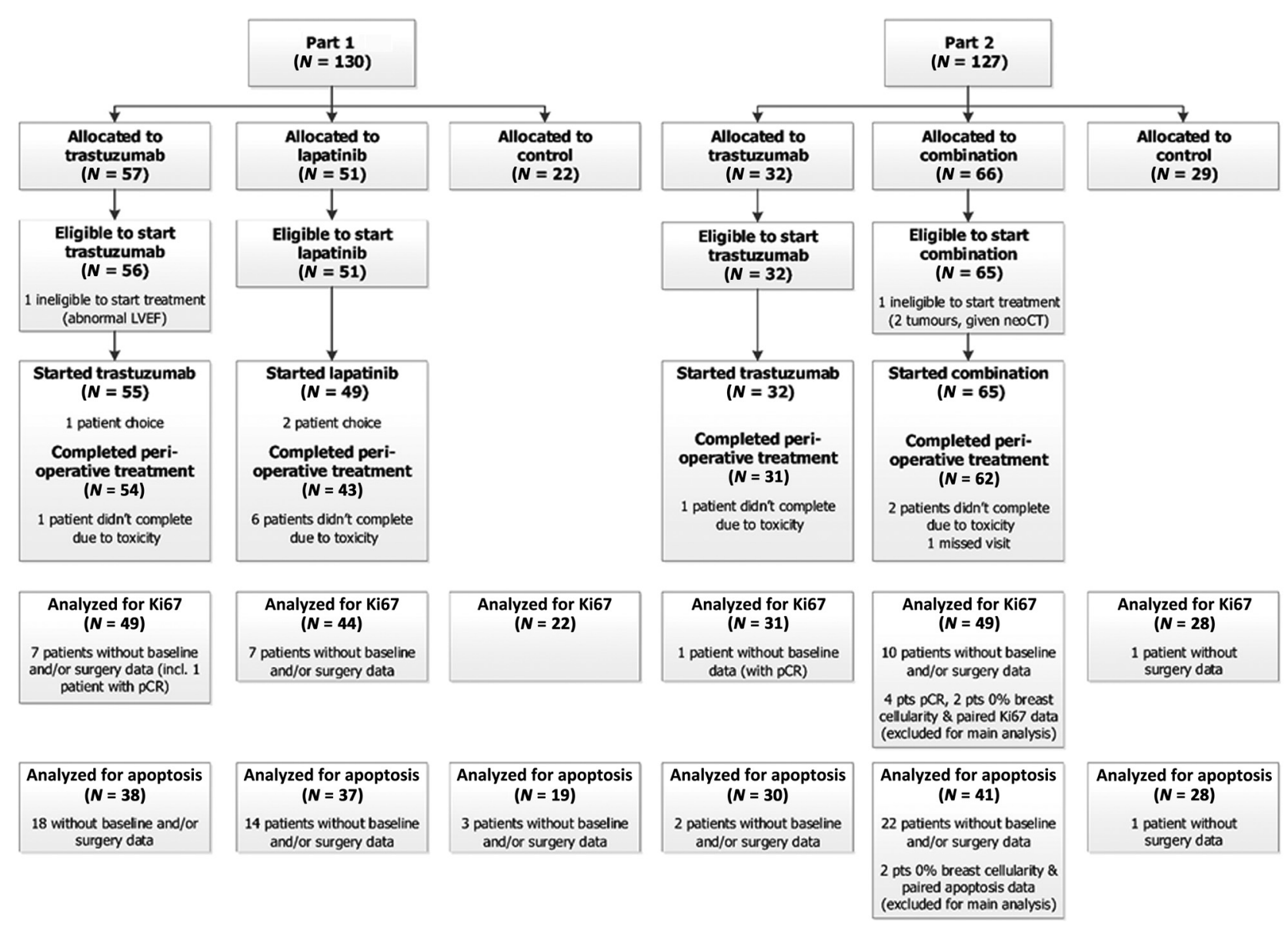

Figure 1.

The CONSORT diagram summarizes patients recruited into each part of the trial, patients randomized, patients eligible to start treatment, patients who started treatment, and those who completed perioperative treatment as per protocol. In part 1, 22 patients were allocated to control, 57 to trastuzumab, and 51 to lapatinib; in part 2, 29 were allocated to control, 32 to trastuzumab, and 66 to the combination. Overall, 255 (99\%) patients were considered eligible to start treatment and included in the analysis of perioperative endpoints. Of the 204 patients in the treatment groups, 201 patients (99\%) received some perioperative treatment, with 190/201 (95\%) completing the 11 days of perioperative treatment. The figure also describes how many patients available for analysis of coprimary endpoints Ki67 and apoptosis. Only patients with both paired samples and enough tumor tissue for biomarker analysis were included in the analysis: 223 patients (88\%) had paired Ki67 data and 193 (76\%) had paired apoptosis data available for analysis. Patients with pCR or 0\% breast cellularity were excluded from main analysis of Ki67 and apoptosis.

patients in the control group $\left(P_{\mathrm{TvC}}<0.001\right)$. Median percentage change in Ki67 was $-20 \%$ (IQR, $-50 \%$ to $+2 \%$ ) with the trastuzumab group and $0 \%$ (IQR, $-13 \%$ to $+11 \%)$ in the control group.

In sensitivity analyses where $0 \%$ Ki67 at surgery was imputed in patients with breast pCR, similar results were obtained (Appendix 4.2). Treatment differences remained after adjusting for known prognostic factors (Appendix 4.3). In exploratory multivariable analyses of the pooled dataset, no other factors (including ER and PgR status) were found to be associated with Ki67 decrease (Appendix 4.4).

HER2 gene amplification ratio (HER2/CEP17 ratio) by FISH (centrally assessed) correlated with change in Ki67 in the trastuzumab group, both in part $2(P=0.008)$, and part 1 and part 2 combined $(P=0.04)$; no association was found between amplification ratio and other trial outcomes (Appendix 5).

\section{Apoptosis}

In part 1,2/37 (5\%) patients in the lapatinib group had an apoptosis response ( $>30 \%$ increase) compared with $7 / 38(18 \%)$ patients in the trastuzumab group $\left(P_{\mathrm{LvT}}=0.15\right)$ and $7 / 19(37 \%)$ patients in the control group $\left(P_{\mathrm{LvC}}=0.01\right)$. Median percentage change in apoptosis was $-25 \%$ (IQR, $-42 \%$ to $+1 \%$ ) with lapatinib, $-5 \%$ (IQR, $-18 \%$ to $+21 \%$ ) with trastuzumab, and $+24 \%$ (IQR, $-10 \%$ to $+57 \%$ ) with control (Table 2).

In part 2, 8/41 (20\%) patients in the combination group had an apoptosis response compared with $11 / 30(37 \%)$ in the trastuzumab group $\left(P_{\mathrm{T}+\mathrm{LvT}}=0.17\right)$ and 10/28 (36\%) in the control group $\left(P_{\mathrm{T}+\mathrm{LvC}}=\right.$ 0.17 ). Median percentage change in apoptosis was $-34 \%$ (IQR, $-56 \%$ to $+10 \%$ ) with combination, $+4 \%$ (IQR, $-32 \%$ to $+48 \%$ ) with trastuzumab, and $-2 \%$ (IQR, $-15 \%$ to $+63 \%$ ) with control (Table 2).

When combining parts 1 and 2, 18/68 (26\%) patients in the trastuzumab group had an apoptosis response compared with $17 / 47$ $(36 \%)$ patients in the control group $\left(P_{\mathrm{TvC}}=0.31\right)$. Median percentage change in apoptosis was $+2 \%$ (IQR, $-28 \%$ to $+36 \%$ ) with trastuzumab and $+6 \%$ (IQR, $-12 \%$ to $+62 \%)$ with control.

Changes from baseline in apoptosis correlated positively with changes in proliferation only in the combination group $(P=0.034$; Appendix 6). 
Table 1. Patient demographics and tumor characteristics at baseline and at surgery, by randomized treatment group.

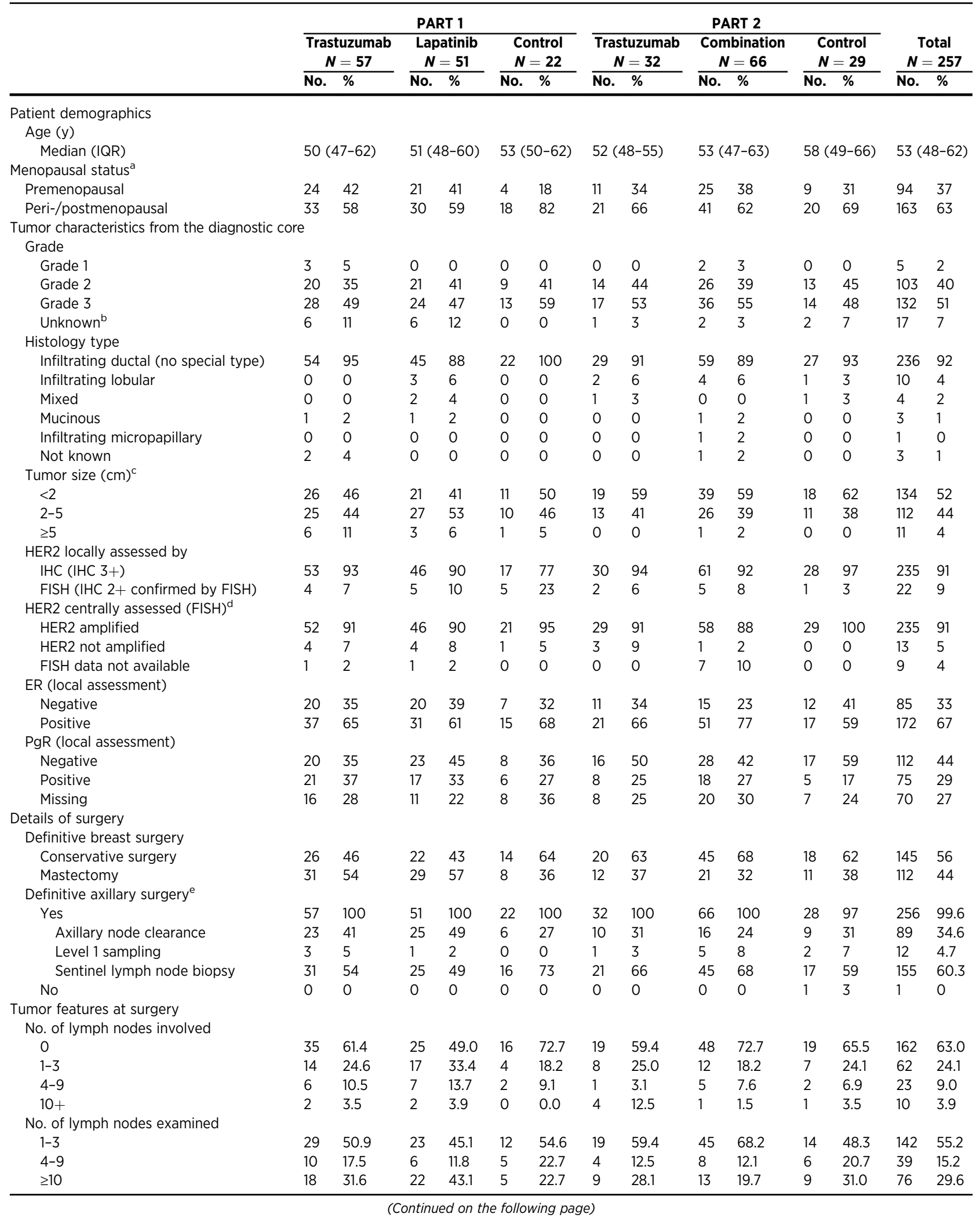


Table 1. Patient demographics and tumor characteristics at baseline and at surgery, by randomized treatment group. (Cont'd)

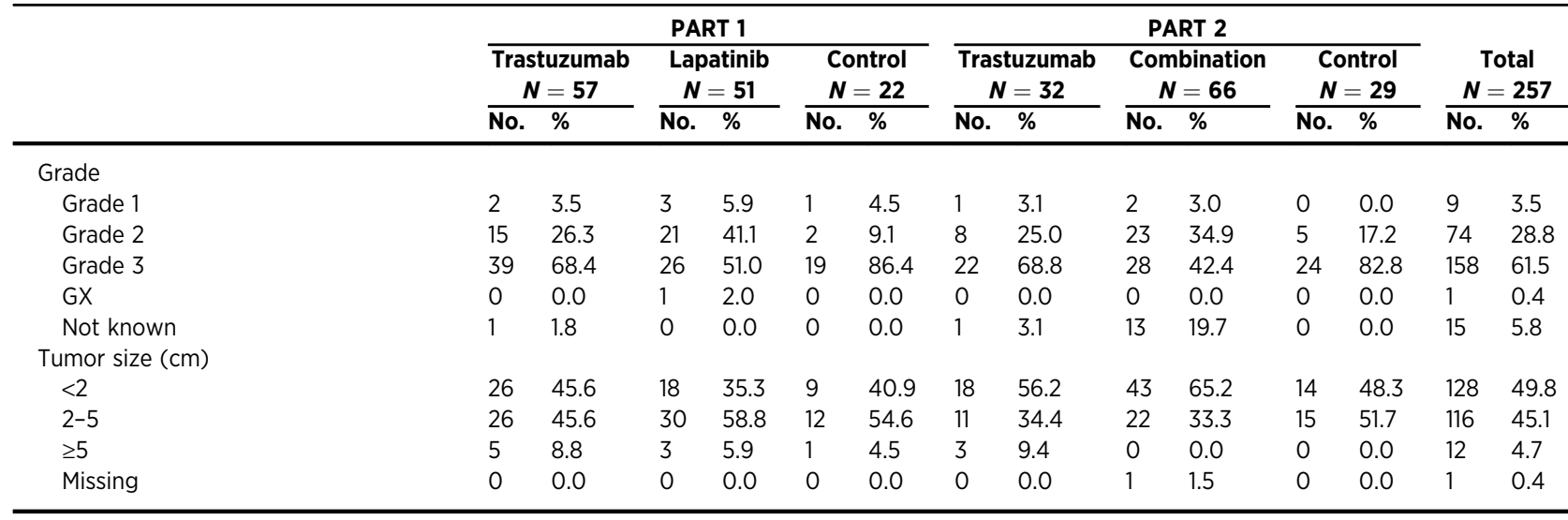

Abbreviations: ER, estrogen receptor; FISH, fluorescence in situ hybridization; HER2, human epidermal growth factor receptor 2; IHC, immunohistochemistry; IQR, interquartile range; PgR, progesterone receptor.

ancludes nine patients (two trastuzumab part 2; five combination part 2; two control part 2) with missing menopausal status data who have been classified on the basis of their age ( $<50=$ premenopausal, $\geq 50=$ peri-/postmenopausal).

${ }^{\mathrm{b}} \mathrm{Some}$ UK hospitals do not routinely report grade on the diagnostic core.

CPresurgery, this measurement is either by ultrasound or clinical examination.

${ }^{d}$ For patients with local FISH testing, scores extracted from pathology reports.

epercentage of mastectomy $(P=0.017)$ and axillary clearance $(P=0.047)$ were found to be lower in part 2 than part 1 .

\section{Five-year time-to-event outcomes}

After median follow-up of 6 years (IQR, 5.2-7.4), 28 women (11\%) had breast cancer recurrence and 19 patients died, with all but one due to breast cancer following recurrence (Appendix 7). The proportion free from breast cancer recurrence at 5 years (5yr-RFS; $95 \% \mathrm{CI})$ was, in part 1, trastuzumab 88\% (76-94), lapatinib 90\% (77-96), and control 95\% (72-99); in part 2, trastuzumab 87\% (69-95), combination $92 \%$ (83-97), and control 90\% (71-97; Fig. 2B). When combining part 1 and part 2, 5yr-RFS were trastuzumab 87\% (79-93) and control 92\% (80-97). There were no significant differences between randomized groups (Fig. 2C), even when adjusting by known prognostic factors (Appendix 7.1), although the study was not powered for such comparisons. Overall survival is shown in Fig. 2D. None of the patients with pCR recurred or died; only one patient in the RCB1 group had an RFS event (local recurrence).

For analysis of perioperative Ki67 changes and RFS, 231/257 patients were included. When categorizing Ki67 change (Fig. 3A), 2/72 (2.8\%) RFS events (local only recurrences, one of these followed by distant recurrence) were observed in the group with Ki67 reductions $\geq 50 \%$, whereas $17 / 77$ (22\%) RFS events (15 distant recurrences, two local only) occurred in the group with reductions between $10 \%$ and $50 \%$, and $7 / 82$ (8.5\%) RFS events (six distant, one local only) were observed in the group with no relevant reduction; RFS was significantly different between the three groups $(P=0.002)$. Such differences remain in multivariable analysis with other prognostic factors (Appendix 7.2). When categorizing absolute Ki67 values, 189 patients (82\%) remained with Ki67 high after 11 days of perioperative treatment ("high-high"), 38 patients (16\%) reduced to low ("high-low"), and 4 (1.7\%) remained low ("low-low"). No patient increased Ki67 from low to high after 11 days of perioperative treatment. Of the 26 RFS events observed, all but one (the local recurrence in a patient with RCB1 response) occurred in the "high-high" group (Fig. 3B).

\section{Exploratory analyses on stromal TILs}

Baseline TILs (bTILs) could be scored for 230/255 patients (90\%); 50 carcinomas (22\%) showed high bTILs $(>20 \%)$; no significant differences were found in bTILs between randomized groups (Appendix 8). We did not observe an association between bTILs and disease response $(P=0.58)$. When associated with RFS (Fig. 3C), 2/50 (4\%) high bTILs experienced an RFS event, versus 23/180 (13\%) among patients with low bTILs $(P=0.06)$.

Ki67 change was $-33 \%$ (IQR, -62 to -8 ) for carcinomas with high bTILs and $-23 \%$ (IQR, -56 to 2$)$ for low bTILs $(P=0.19)$. In the trastuzumab group, Ki67 responses were observed in 8/13 (62\%) high bTILs versus $23 / 62$ (37\%) low bTILs ( $P=0.10$, Appendix 8$)$.

Change from baseline TILs at surgery was calculated in 191/236 (81\%) RCB2/3 patients (Appendix 8). The TILs increase was $\geq 20 \%$ observed in 38/69 (20\%) trastuzumab, 16/43 (23\%) lapatinib, 12/33 (36\%) combination, and in $1 / 46(2 \%)$ control patients $(P=0.002)$. Ki67 response was observed in $21 / 35(60 \%)$ patients with $\geq 20 \%$ TILs increase and in $56 / 152(37 \%)$ patients without $(P=0.012)$. Having high TILs at surgery seemed to explain improved PFS $(P=0.02$, Fig. 3D) rather than having a $\geq 20 \%$ TILs increase between baseline and surgery $(P=0.16)$.

\section{Safety}

Sixteen serious adverse events were reported in 14/257 (5\%) patients. Six were unrelated to treatment [four allocated trastuzumab alone (part 1 and part 2), and two allocated combination]. Ten were classed as expected serious adverse reactions, occurring in two patients allocated to trastuzumab (part 1 and part 2), five allocated to lapatinib (part 1), and three allocated to combination treatment.

An additional cardiac assessment after treatment but before adjuvant chemotherapy was introduced as of April 2014, affecting 90/127 part 2 patients. The assessment was done on 70/90 part 2 patients and one trastuzumab patient showed an abnormal LVEF of $35 \%$, leading to treatment delay. Further details on safety can be found in Appendix 9 .

\section{Discussion}

The EPHOS- $\mathrm{B}$ Trial met one of its primary objectives, that 11 days of anti-HER2 therapy, between diagnosis and surgery, without 
A

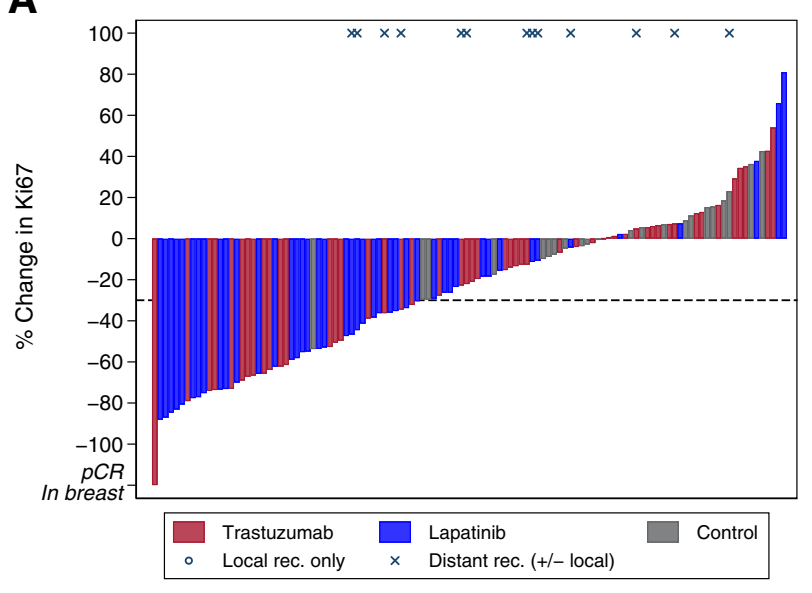

C

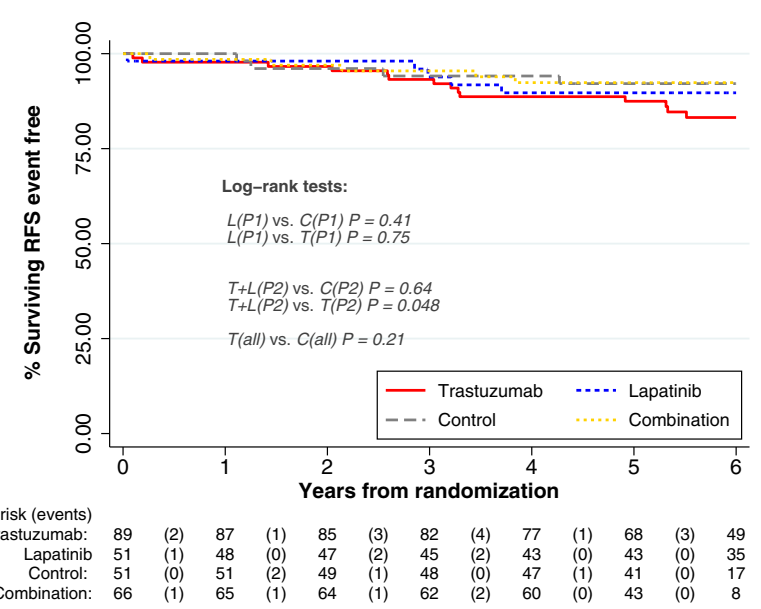

B

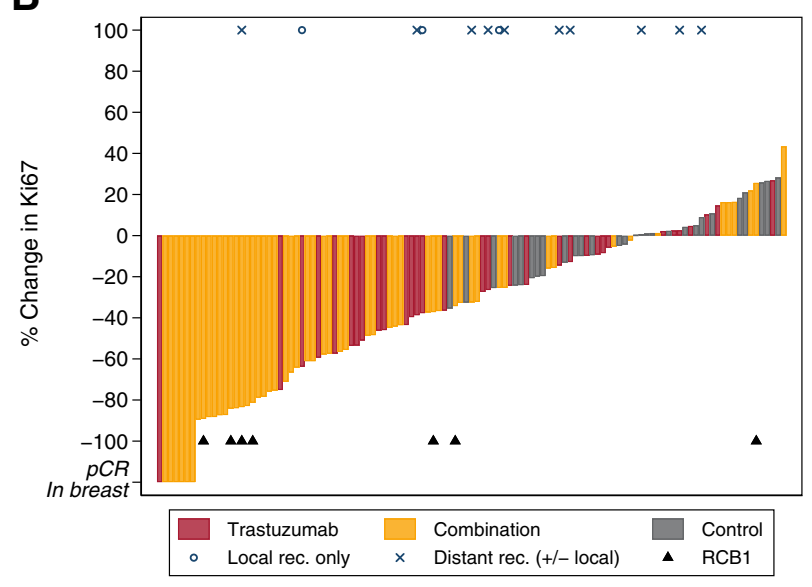

D

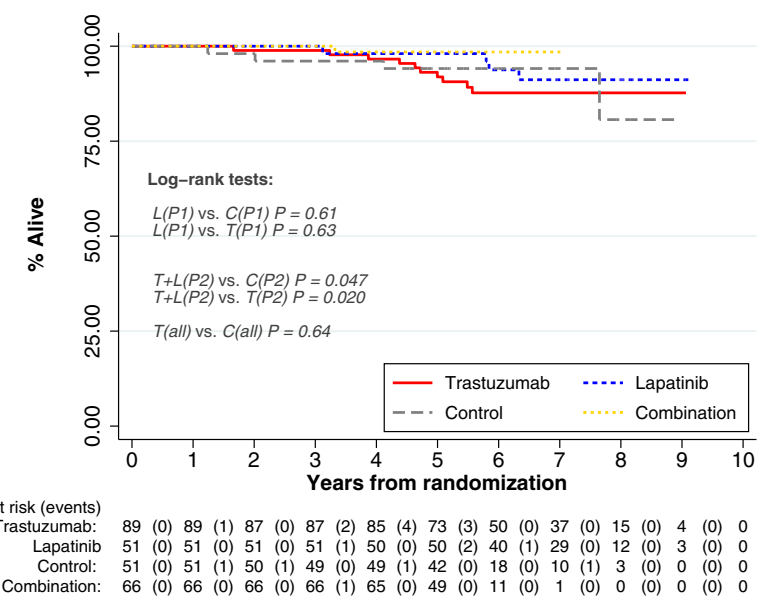

Figure 2.

Percentage change in Ki67 between pretreatment (baseline) and surgery for part 1 (A) and part 2 (B); Kaplan-Meier estimates by treatment group for relapse free survival (C) and overall survival (D). A, Waterfall plots for part 1 and part 2: for each patient, bar height represents percentage change at surgery from baseline. Percentage change was calculated as [(surgery score +0.1$)-($ pretreatment score +0.1$)] /[(\text { pretreatment score }+0.1)]^{*} 100$. The constant of 0.1 was added to accommodate cases with a value of $0 \%$. Negative values represent decrease from baseline, positive values represent increase from baseline. pCR in breast: patients with pCR (no disease in ether breast or nodes) plus two additional patients with $0 \%$ breast cellularity but nodal involvement are represented as bars of height -120\% at the left of the figures and noted " $p C R$ in breast;" any existing Ki67 values for these patients have been excluded of the main analysis; in a sensitivity analysis, we imputed a value of $-100 \%$ change for these patients (Appendix 2). Small triangles indicate patients with RCB1. Disease recurrences are also indicated at the top of each figure with circles and crosses. B, RFS is represented in the time interval of up to 6 years after randomization, as no RFS event occurred later. Overall survival is represented in the fully observed range of values. Log-rank test comparing concurrently randomized treatment groups are reported in the figures. In the figure, trastuzumab and control part 1 and part 2 groups are combined to improve readability. C, control; L, lapatinib; T, trastuzumab; T+L, combination; P1, part 1; P2, part 2; all, P1\&P2; $P, P$ value.

chemotherapy, reduced proliferation, which was seen in all active treatment groups but particularly with the dual-agent combination where a Ki67 decrease greater than $30 \%$ was seen in $74 \%$ of cancers. Furthermore, some tumors became too small to be analyzed at the time of surgery, and exploratory analysis revealed dual blockade with lapatinib and trastuzumab resulted in 4/65 (6\%) of cases having no residual invasive disease in the breast or nodes (pCR) and a further 13/ $65(20 \%)$ cases with only minimal residual disease (RCB1).

The EPHOS-B trial did not meet its second primary objective of showing an increase in apoptosis in treatment groups, in contrast to a small clinical study that reported increases in apoptosis after 7 days after beginning treatment (16), so potentially, because the ontreatment assessment was performed after 11 days of treatment, in some patients any increase in apoptosis may have been missed.
Furthermore, high proliferation values at baseline correlated with higher apoptosis, and, in treatment groups, the fall in proliferation led to a fall in apoptosis, as observed elsewhere $(6,18)$, so few treated patients had a 30\% increase in apoptosis. Although the baseline core biopsies had high numbers of Ki67-positive cells to count, low values of apoptosis rose in control patients due to the greater accuracy of assessment on the surgical excision specimens, but fell with the antiproliferative effect of treatments (19).

Both trastuzumab and lapatinib have been previously shown to inhibit HER2-positive breast cancer proliferation when given before surgery $(1,2,4)$. Changes in proliferation biomarkers, including Ki67, predict clinical response and long-term outcome after 2 weeks of endocrine therapy in ER-positive breast cancer (5-7). In our study, several safeguards were in place to enable exploratory associations of 


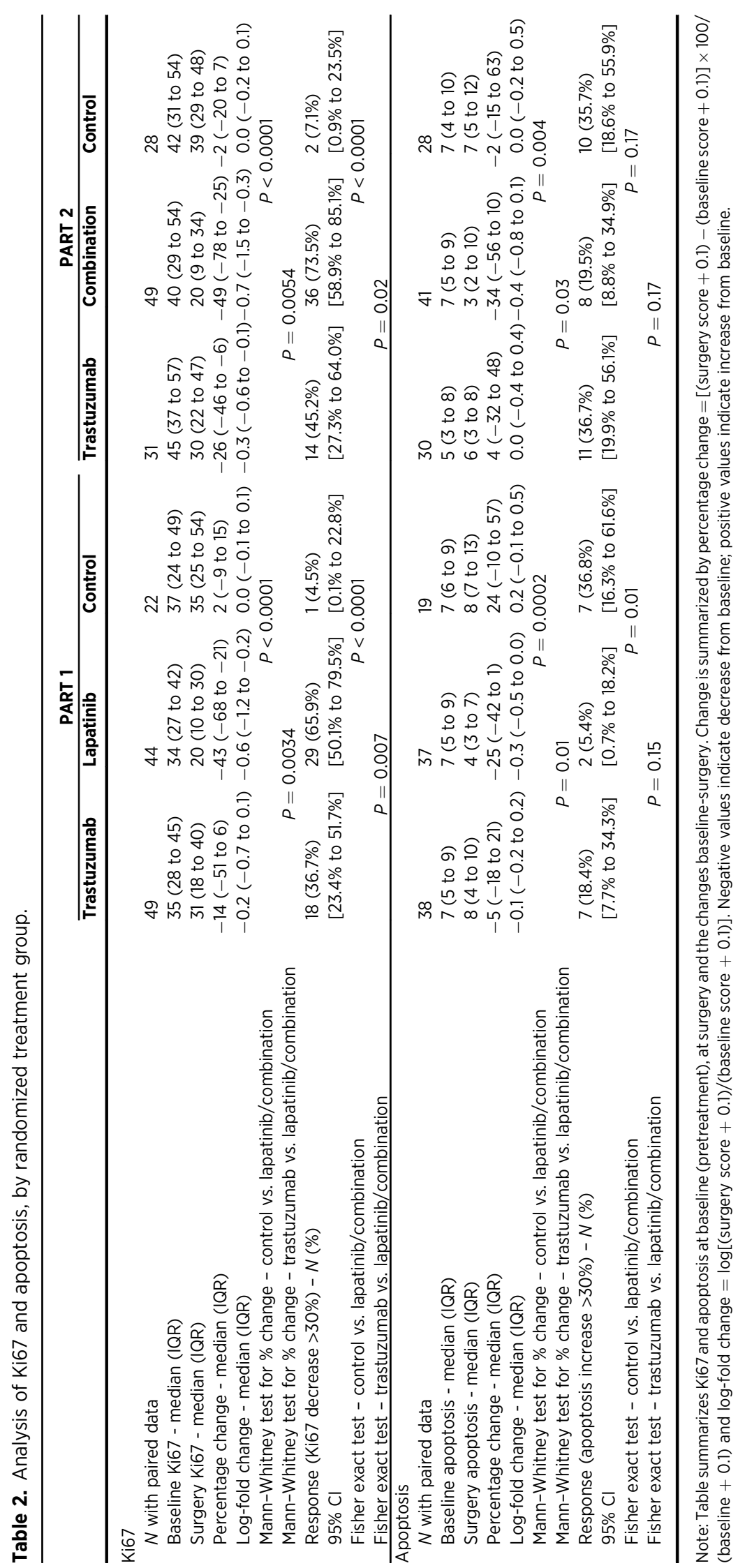


A

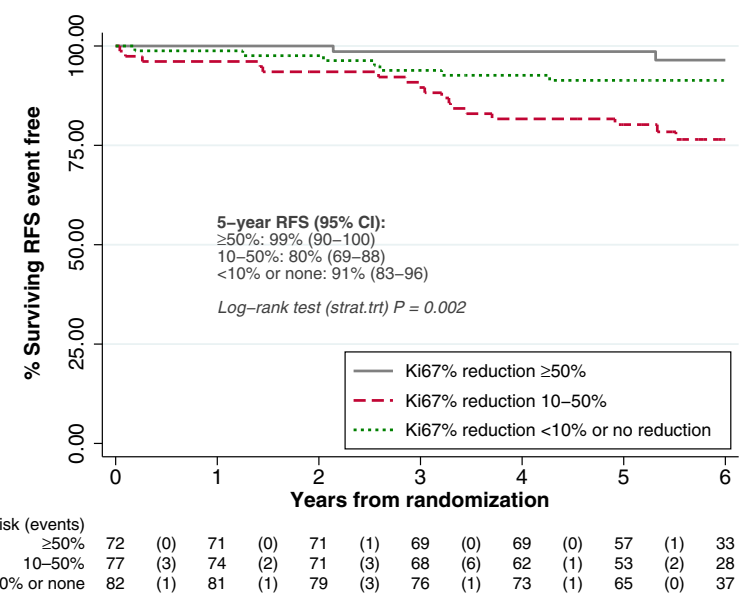

C

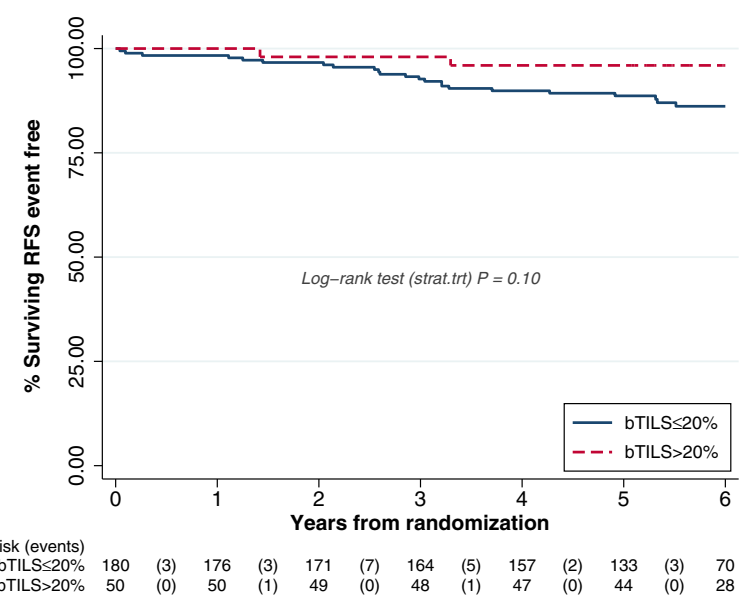

B

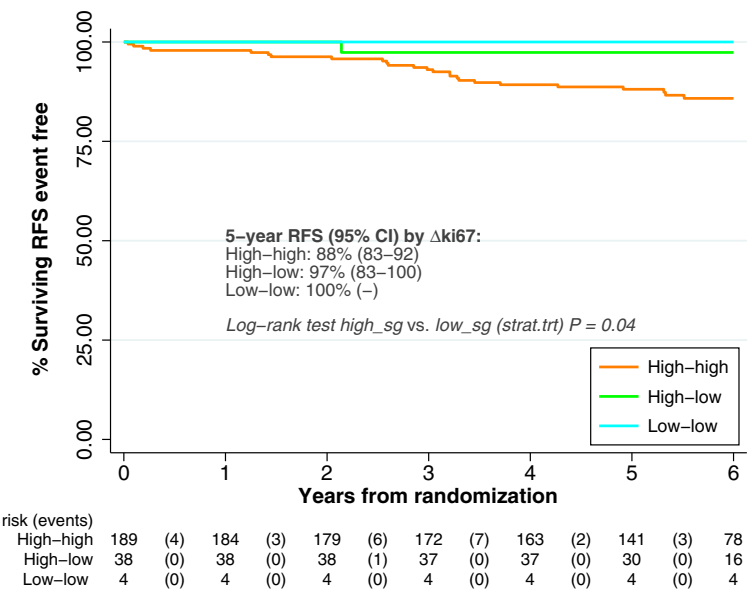

D

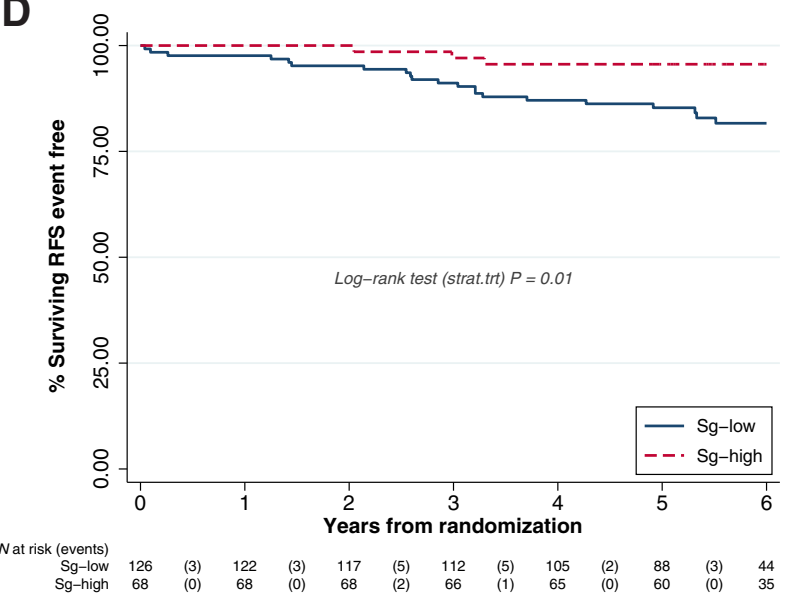

Figure 3.

Association of perioperative changes in biological markers with RFS (A) by categories of Ki67 relative change, (B) by categories of Ki67 absolute change, (C) by baseline TILs, (D) by surgery TILs. RFS is represented in the time interval 0 to 6 years, as no RFS events occurred beyond 6 years from randomization. All treatment groups are combined; log-rank tests are stratified by treatment group $(P=P$ value). For $\mathbf{A}$ and $\mathbf{B}$, a value of $-100 \%$ Ki67 change $(\Delta \mathrm{Ki}$ (67) has been imputed for patients with a pCR in breast. For $\mathbf{B}$, we have categorized both baseline and surgery Ki67 into high if $\geq 10 \%$ or low if $<10 \%$. No patient increased Ki67 from low to high after 11 days of perioperative treatment. Because of small number of patients in the "low-low" group, we have compared patients with "high" value at surgery with patients with "low" value at surgery.

biomarkers with long-term outcomes: adjuvant therapy was to be given as per local protocols; Ki67 results were not fed back to investigators; and centralized review of RCB status was done retrospectively. Although adjuvant treatment may be a confounding factor for long-term outcomes, we did not observe differences in adjuvant treatment received between ki67 change groups (see Appendix 2), nor that RCB0 or RCB1 responders received any more or less treatment (see Appendix 3). In these exploratory analyses, patients with Ki67 reductions $\geq 50 \%$ at 11 days had 5 -year RFS $99 \%$.

Reporting eradication of primary tumors after only 11 days' dual anti-HER2 blockade therapy is unprecedented. In the NEO-SPHERE trial (9), 18/107 (17\%) patients achieved pCR after 4 months' treatment with pertuzumab and trastuzumab and no chemotherapy; for ER and/or PgR-positive tumors, only $3 / 51(6 \%)$ achieved pCR. In the TBCRC006 study (1), patients received lapatinib and trastuzumab, with ER-positive patients (62\%) also receiving letrozole. After 12 weeks' therapy, 17/64 (27\%) patients achieved pCR: 8/39 (21\%) among ER-positive, 9/25 (36\%) among ER-negative. In the WSG-ADAPT study (20), 12-week treatment of HER2-positive/ER-negative cancers with trastuzumab and pertuzumab (without chemotherapy) led to 74\% exhibiting Ki67 reductions $\geq 30 \%$, and $36 \%$ pCR. Ki67 nonresponders had an $8 \%$ pCR rate. It is worth noting that for neo-adjuvant HER2 trials, tumors were typically over $2 \mathrm{~cm}$ in size at trial entry, whereas this was not a requirement in EPHOS-B, and the chance of achieving PCR in the combination group was lower for larger tumors.

In EPHOS-B, 26\% combination patients whose cancers regressed (pCR or RCB1) was consistent with $30 \%$ pCR seen in the PAMELA study (21) and 27\% pCR in TBCRC006 (1) after 12 weeks of neoadjuvant dual agent therapy. EPHOS-B used a trastuzumab-accelerated loading dose (6 mg/kg; ref. 22) combined with lapatinib 1,000 mg to ensure maximal HER 2 blockade by 11 days, which may partly account for the earlier responses, as previous neoadjuvant studies used a lower initial doses of trastuzumab $(9,21,23)$

Imaging substudies assessing (18)F-FDG PET/CT at 15 days in the Neo-ALTTO and TBCRC026 trials showed greater $\mathrm{SUV}_{\max }$ reductions predicted pCR in response to dual anti-HER2 therapy $(24,25)$. However PET/CT studies are not widely available for clinical practice, whereas Ki67 and tumor response at 11 days are practical for wider 
implementation in the neoadjuvant setting to predict response on dual anti-HER2 therapy potentially reducing toxicity.

ER and HER2-positive tumors are less likely than ER-negative tumors to have pCR in response to several months of anti-HER2 therapy $(9,23,26-28)$. Observing $6 \%$ pCR and $20 \%$ RCB1 in a population with two-thirds having ER-positive tumors, after 11 days' therapy, was a surprising finding, as there was no evidence that the ER status of the cancer influenced pCR. pCR incidence in ER-positive/ HER2-positive cancers is usually lower than in ER-negative/HER2positive cancers (29). The observed effects on both Ki67 and tumor response in the ER- and HER2-positive cancers may relate to the second anti-HER2 therapy used. Tyrosine kinase inhibitors such as lapatinib interfere with the intercellular tyrosine kinase signaling, which is known to interact with ER signaling. Recent pCR reports in $27 \%$ to $44 \%$ HER2-positive cancers after 12 to 16 weeks of combination therapy $(30,31)$ imply that there is a group of patients with HER2 whose primary cancers are highly dependent on HER2 signaling, and their early identification would (if validated in further studies) allow testing of the omission of chemotherapy without detrimental effects on oncologic outcomes.

The WSG-ADAPT (20) trial tested a de-escalation approach following identification of early responders: in largely stage 1 HER2positive HR-negative breast cancers, after Ki67 assessment at 3 weeks of pertuzumab and trastuzumab, patients were randomized to continued dual agent therapy up to 12 weeks, or combined it with weekly paclitaxel. Nonresponse at 3 weeks predicted lack of pCR at 12 weeks, but addition of paclitaxel in early responders produced $79 \%$ pCR, superior to $45 \%$ pCR observed when no paclitaxel was added.

The PerELISA (32) neoadjuvant study enrolled mainly patients with stage 2/3 HR-negative HER2-positive cancers that were treated for 2 weeks with an aromatase inhibitor and re-biopsied. Reductions in Ki67 $\geq 50 \%$ allowed treatment with dual-agent pertuzumab and trastuzumab, whereas nonresponders additionally received paclitaxel. After 13 weeks of treatment, pCR and RCB1 occurred in 52\% of early responders. If dual antibody therapy can eradicate some HER2positive breast cancers in less than 2 weeks, a similar approach using a letrozole and dual anti-HER2 therapy from initial biopsy may improve selection of patients who can avoid chemotherapy. These studies imply that a Ki67 reduction $>50 \%$ to anti-HER2 therapy after 2 weeks of treatment predicts outcome and that approaches to deescalation will likely differ according to ER status. Our data, taken with these studies, suggest there may be HER2-positive breast cancers that can be eradicated without chemotherapy. Our data add evidence that reductions in Ki67 or pCR/RCB1 (by image-guided biopsy or surgery) after 11 days of treatment potentially allows clinicians to select patients who could receive less chemotherapy, a strategy that needs validation in further studies.

TILs affect RFS and response to therapy, but the effect seems driven by trastuzumab (alone or in an combination): among RCB $2 / 3$ trastuzumab patients, a higher Ki67 response was observed when a relevant increase in TILs occurred (62\%) compared with those without an increase in TILs (43\%, Appendix 7). Moreover, the phenotype of TILs may also alter from suppressor to effector TILs, but we could not assess that on the samples available. The alterations in TILs $>20 \%$ with the large reduction in tumor proliferation may have produced the tumor shrinkage seen by 11 days.

Early data with trastuzumab, when given concurrently with or after adjuvant chemotherapy, led to concerns about cardiotoxicity, (33) but there were no effects on LVEF in the combination group, and no operations were rescheduled because of cardiac issues in our study. All three neoadjuvant trials using dual-agent HER2 blockade with chemotherapy have not found increased short-term cardiotoxicity. $(2,9,23)$ A significant part of the cardiac toxicity reported with anti-HER2 therapy may relate to its use with anthracycline chemotherapy.

The next generation of studies of anti-HER2 therapy in early breast cancer need to address both the potential to reduce chemotherapy in some patients and additional approaches in others, as defined by their demonstrated sensitivity to short-duration anti-HER2 therapies. The data we report on the early disappearance of tumors 11 days after treatment commencement may identify a patient group highly sensitive to the HER2-pathway who can potentially avoid chemotherapy altogether.

\section{Authors' Disclosures}

A.M. Shaaban reports personal fees from Exact Sciences Advisory Board, Veracyte Advisory Board, AstraZeneca Advisory Board, and Daiichi Sankyo Advisory Board during the conduct of the study. A.C. Armstrong reports other support from AstraZeneca, grants from AstraZeneca, personal fees from Gilead and Merck Sharp \& Dohme, and nonfinancial support from Gilead and Merck Sharp \& Dohme outside the submitted work. R.I. Cutress reports grants from AstraZeneca and nonfinancial support from Seca outside the submitted work. S.A. McIntosh reports personal fees and nonfinancial support from Roche Products Limited outside the submitted work. D.A. Cameron reports other support from GlaxoSmithKline, grants and other support from Novartis and Roche, and other support from Syntheon and SeaGen outside the submitted work. J.M. Bliss reports grants from AstraZeneca, Merck Sharp \& Dohme, Puma Biotechnology, Clovis Oncology, Pfizer, JanssenCilag, Novartis, Roche, and Eli Lilly outside the submitted work. No disclosures were reported by the other authors.

\section{Authors' Contributions}

N. Bundred: Conceptualization, resources, data curation, supervision, funding acquisition, investigation, visualization, methodology, writing-original draft, writing-review and editing. N. Porta: Data curation, software, formal analysis, investigation, visualization, writing-original draft, writing-review and editing. A.M. Brunt: Resources, investigation, writing-review and editing. A. Cramer: Resources, data curation, investigation, writing-review and editing. A. Hanby: Conceptualization, resources, data curation, funding acquisition, investigation, writing-review and editing. A.M. Shaaban: Resources, data curation, investigation, writing-review and editing. E.A. Rakha: Resources, data curation, investigation, writing-review and editing. A. Armstrong: Resources, writing-review and editing. R.I. Cutress: Resources, investigation, writing-review and editing D. Dodwell: Resources, writing-review and editing. M.A. Emson: Conceptualization, investigation, project administration, writing-review and editing. A. Evans: Resources, investigation, writing-review and editing. S.M. Hartup Resources, investigation, writing-review and editing. K. Horgan: Resources, investigation, writing-review and editing. S.E. Miller: Investigation, project administration, writing-review and editing. S.A. McIntosh: Resources, investigation, writing-review and editing. J.P. Morden: Data curation, software, formal analysis, investigation, visualization, writing-original draft. J. Naik: Resources, investigation, writing-review and editing. S. Narayanan: Resources, writingreview and editing. J. Ooi: Resources, writing-review and editing. A.I. Skene Resources, investigation, writing-review and editing. D.A. Cameron: Conceptualization, resources, supervision, funding acquisition, investigation, writingoriginal draft, writing-review and editing. J.M. Bliss: Conceptualization, resources, data curation, software, formal analysis, supervision, funding acquisition, investigation, visualization, writing-original draft, project administration, writing-review and editing.

\section{Acknowledgments}

We thank all participating patients and their families, involved staff at participating hospitals, and staff involved in the trial at The Institute of Cancer Research Clinical Trials \& Statistics Unit (ICR-CTSU). The trial was funded by Cancer Research UK with approval of its design from their Clinical Trials Awards and Advisory Committee (CRUK/08/002). ICR-CTSU receives programme gran funding from Cancer Research UK (grant Nos. C1491/A9895, C1491/A15955, C1491/A25351). Lapatinib was provided to centers by Novartis (formerly GlaxoSmithKline), who also supplied an educational grant. None of the funders 
had a direct role in study design, data collection, data analysis, data interpretation, or writing of the report.

The EPHOS-B trial represents independent research supported by the National Institute for Health Research (NIHR) Biomedical Research Centre at The Royal Marsden NHS Foundation Trust and the Institute of Cancer Research, London. The views expressed are those of the authors and not necessarily those of the NIHR or the Department of Health and Social Care. We acknowledge support at participating sites by the NIHR Cancer Research Network (CRN)/NHS Research Scotland/Health and Care Research Wales.

Finally, we thank the past and present colleagues on the EPHOS-B Trial Management Group, the EPHOS-B Translational Sub-Committee, the ICR-CTSU Breast Systemic Trials Steering Committee, and the EPHOS-B

\section{References}

1. Rimawi MF, Mayer IA, Forero A, Nanda R, Goetz MP, Rodriguez AA, et al. Multicenter phase II study of neoadjuvant lapatinib and trastuzumab with hormonal therapy and without chemotherapy in patients with human epidermal growth factor receptor 2-overexpressing breast cancer: TBCRC 006. J Clin Oncol 2013;31:1726-31.

2. Guarneri V, Dieci MV, Frassoldati A, Maiorana A, Ficarra G, Bettelli S, et al. Prospective biomarker analysis of the randomized CHER-LOB Study evaluating the dual Anti-HER2 treatment with trastuzumab and lapatinib plus chemotherapy as neoadjuvant therapy for HER2-positive breast cancer. Oncologist 2015;20:1001-10.

3. Kumler I, Tuxen MK, Nielsen DL. A systematic review of dual targeting in HER2positive breast cancer. Cancer Treat Rev 2014;40:259-70.

4. Leary A, Evans A, Johnston SRD, A'Hern R, Bliss JM, Sahoo R, et al Antiproliferative effect of lapatinib in HER2-positive and HER2-negative/ HER3-high breast cancer: results of the presurgical randomized MAPLE Trial (CRUK E/06/039). Clin Cancer Res 2015;21:2932-40.

5. Dowsett M, Bundred NJ, Decensi A, Sainsbury RC, Lu Y, Hills MJ, et al. Effect of raloxifene on breast cancer cell Ki67 and apoptosis: a double-blind, placebo-controlled, randomized clinical trial in postmenopausal patients. Cancer Epidemiol Biomarkers Prev 2001;10:961-6.

6. Dowsett M, Smith IE, Ebbs SR, Dixon JM, Skene A, Griffith C, et al. Short-term changes in Ki-67 during neoadjuvant treatment of primary breast cancer with anastrozole or tamoxifen alone or combined correlate with recurrence-free survival. Clin Cancer Res 2005;11:951s-8s.

7. Smith I, Robertson J, Kilburn L, Wilcox M, Evans A, Holcombe C, et al. Longterm outcome and prognostic value of Ki67 after perioperative endocrine therapy in postmenopausal women with hormone-sensitive early breast cancer (POETIC): an open-label, multicentre, parallel-group, randomised, phase 3 trial. Lancet Oncol 2020;21:1443-54.

8. Tagliabue E, Agresti R, Carcangiu ML, Ghirelli C, Morelli D, Campiglio M, et al. Role of HER2 in wound-induced breast carcinoma proliferation. Lancet 2003; 362:527-33.

9. Gianni L, Pienkowski T, Im Y-H, Roman L, Tseng L-M, Liu M-C, et al. Efficacy and safety of neoadjuvant pertuzumab and trastuzumab in women with locally advanced, inflammatory, or early HER2-positive breast cancer (NeoSphere): a randomised multicentre, open-label, phase 2 trial. Lancet Oncol 2012;13:25-32.

10. Leyland-Jones B, Colomer R, Trudeau ME, Wardley A, Latreille J, Cameron $\mathrm{D}$, et al. Intensive loading dose of trastuzumab achieves higher-thansteady-state serum concentrations and is well tolerated. J Clin Oncol 2010;28:960-6.

11. Hadjiloucas I, Gilmore AP, Bundred NJ, Streuli CH. Assessment of apoptosis in human breast tissue using an antibody against the active form of caspase 3 : relation to tumour histopathological characteristics. Br J Cancer 2001;85: 1522-6.

12. Wolff AC, Hammond MEH, Hicks DG, Dowsett M, McShane LM, Allison $\mathrm{KH}$, et al. Recommendations for human epidermal growth factor receptor 2 testing in breast cancer: American Society of Clinical Oncology/College of American Pathologists clinical practice guideline update. J Clin Oncol 2013; 31:3997-4013.

13. Rakha EA, Pinder SE, Bartlett JMS, Ibrahim M, Starczynski J, Carder PJ, et al Updated UK recommendations for HER2 assessment in breast cancer. J Clin Pathol 2015;68:93-9.

14. Salgado R, Denkert C, Demaria S, Sirtaine N, Klauschen F, Pruneri G, et al. The evaluation of tumor-infiltrating lymphocytes (TILs) in breast cancer:
Independent Data Monitoring Committee. EPHOS-B is cosponsored by University of Manchester, Manchester University NHS Foundation Trust (formerly University Hospital of South Manchester NHS Foundation Trust) and The Institute of Cancer Research.

The costs of publication of this article were defrayed in part by the payment of page charges. This article must therefore be hereby marked advertisement in accordance with 18 U.S.C. Section 1734 solely to indicate this fact.

Received September 1, 2021; revised November 11, 2021; accepted January 20, 2022; published first February 14, 2022.

recommendations by an International TILs Working Group Ann Oncol 2015;26:259-71.

15. Provenzano E, Bossuyt V, Viale G, Cameron D, Badve S, Denkert C, et al. Standardization of pathologic evaluation and reporting of postneoadjuvant specimens in clinical trials of breast cancer: recommendations from an international working group. Mod Pathol 2015;28:1185-201.

16. Mohsin SK, Weiss HL, Gutierrez MC, Chamness GC, Schiff R, DiGiovanna MP, et al. Neoadjuvant trastuzumab induces apoptosis in primary breast cancers. J Clin Oncol 2005;23:2460-8.

17. Luen SJ, Salgado R, Fox S, Savas P, Eng-Wong J, Clark E, et al. Tumourinfiltrating lymphocytes in advanced HER2-positive breast cancer treated with pertuzumab or placebo in addition to trastuzumab and docetaxel: a retrospective analysis of the CLEOPATRA study. Lancet Oncol 2017;18: $52-62$.

18. Johnston S, Puhalla S, Wheatley D, Ring A, Barry P, Holcombe C, et al. Randomized phase II study evaluating palbociclib in addition to letrozole as neoadjuvant therapy in estrogen receptor-positive early breast cancer: PALLET trial. J Clin Oncol 2019;37:178-89.

19. Green DR, Evan GI. A matter of life and death. Cancer Cell 2002;1:19-30.

20. Nitz UA, Gluz O, Christgen M, Grischke EM, Augustin D, Kuemmel S, et al. Deescalation strategies in HER2-positive early breast cancer (EBC): final analysis of the WSG-ADAPT HER2+/HR - phase II trial: efficacy, safety, and predictive markers for 12 weeks of neoadjuvant dual blockade with trastuzumab and pertuzumab \pm weekly paclitaxel. Ann Oncol 2017;28:2768-72.

21. Llombart-Cussac A, Cortés J, Paré L, Galván P, Bermejo B, Martínez N, et al. HER2-enriched subtype as a predictor of pathological complete response following trastuzumab and lapatinib without chemotherapy in early-stage HER2-positive breast cancer (PAMELA): an open-label, single-group, multicentre, phase 2 trial. Lancet Oncol 2017;18:545-54.

22. Leyland-Jones B, Gelmon K, Ayoub J-P, Arnold A, Verma S, Dias R, et al. Pharmacokinetics, safety, and efficacy of trastuzumab administered every three weeks in combination with paclitaxel. J Clin Oncol 2003;21: 3965-71.

23. Baselga J, Bradbury I, Eidtmann H, Di Cosimo S, de Azambuja E, Aura C, et al. Lapatinib with trastuzumab for HER2-positive early breast cancer (NeoALTTO): a randomised, open-label, multicentre, phase 3 trial. Lancet 2012;379:633-40.

24. Gebhart G, Gámez C, Holmes E, Robles J, Garcia C, Cortés M, et al. 18F-FDG $\mathrm{PET} / \mathrm{CT}$ for early prediction of response to neoadjuvant lapatinib, trastuzumab, and their combination in HER2-positive breast cancer: results from NeoALTTO. J Nucl Med 2013;54:1862-8.

25. Connolly RM, Leal JP, Solnes L, Huang C-Y, Carpenter A, Gaffney K, et al. TBCRC026: Phase II Trial correlating standardized uptake value with pathologic complete response to pertuzumab and trastuzumab in breast cancer. J Clin Oncol 2019;37:714-22.

26. Park S, Jiang Z, Mortenson ED, Deng L, Radkevich-Brown O, Yang X, et al. The therapeutic effect of anti-HER2/neu antibody depends on both innate and adaptive immunity. Cancer Cell 2010;18:160-70.

27. Chan A, Delaloge S, Holmes FA, Moy B, Iwata H, Harvey VJ, et al. Neratinib after trastuzumab-based adjuvant therapy in patients with HER2-positive breast cancer (ExteNET): a multicentre, randomised, double-blind, placebo-controlled, phase 3 trial. Lancet Oncol 2016;17:367-77.

28. Clavarezza M, Puntoni M, Gennari A, Paleari L, Provinciali N, D’Amico M, et al. Dual block with lapatinib and trastuzumab versus single-agent trastuzumab combined with chemotherapy as neoadjuvant treatment of HER2- 
Bundred et al.

positive breast cancer: a meta-analysis of randomized trials. Clin Cancer Res 2016;22:4594-603.

29. Esserman LJ, Berry DA, DeMichele A, Carey L, Davis SE, Buxton M, et al. Pathologic complete response predicts recurrence-free survival more effectively by cancer subset: results from the I-SPY 1 TRIAL-CALGB 150007/150012, ACRIN 6657. J Clin Oncol 2012;30:3242-9.

30. Gianni L, Bisagni G, Colleoni M, Del Mastro L, Zamagni C, Mansutti M, et al. Neoadjuvant treatment with trastuzumab and pertuzumab plus palbociclib and fulvestrant in HER2-positive, ER-positive breast cancer (NA-PHER2): an exploratory, open-label, phase 2 study. Lancet Oncol 2018;19:249-56.
31. Hurvitz SA, Martin M, Symmans WF, Jung KH, Huang C-S, Thompson AM et al. Neoadjuvant trastuzumab, pertuzumab, and chemotherapy versus trastuzumab emtansine plus pertuzumab in patients with HER2-positive breast cance (KRISTINE): a randomised, open-label, multicentre, phase 3 trial. Lancet Onco 2018;19:115-26.

32. Guarneri V, Dieci MV, Bisagni G, Frassoldati A, Bianchi GV, De Salvo GL, et al. De-escalated therapy for HR+/HER2+ breast cancer patients with Ki67 response after 2-week letrozole: results of the PerELISA neoadjuvant study. Ann Oncol 2019;30:921-6.

33. Valachis A, Nearchou A, Polyzos NP, Lind P. Cardiac toxicity in breast cancer patients treated with dual HER2 blockade. Int J Cancer 2013;133:2245-52. 